\title{
ARTICLE
}

\section{Performance of the beam collimation system in the J-PARC RCS}

\author{
Kazami Yamamoto ${ }^{a^{*}}$, Shinichi Kato ${ }^{\mathrm{b}}$, Hiroyuki Harada ${ }^{\mathrm{a}}$, Masahiro Yoshimoto ${ }^{\mathrm{a}}$, Yoshio Yamazaki ${ }^{\mathrm{a}}$, Naoki Hayashi ${ }^{\mathrm{a}}$, \\ Pranab Kumar Saha ${ }^{\mathrm{a}}$, Hideaki Hotchi ${ }^{\mathrm{a}}$ and Michikazu Kinsho ${ }^{\mathrm{a}}$

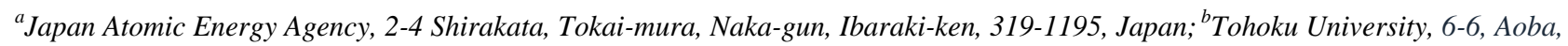 \\ Aramaki, Aoba-ku, Sendai, 980-0872, Japan
}

\begin{abstract}
The most important issue in a high intensity proton accelerator is to keep the accelerator tunnel in a hands-on-maintenance condition. A collimation system was designed and installed in $3 \mathrm{GeV}$ Rapid Cycling Synchrotron (RCS) at the Japan Proton Accelerator Research Complex (J-PARC) to be localized the beam loss point to a restricted area. The experimental results indicated that the RCS collimation system performs well enough such that nearly all the ring components are maintained in good condition. However, as the beam power increased, unexpected losses downstream of the injection foil increased. Therefore, an additional collimator was installed that successfully reduced such unexpected losses.
\end{abstract}

Keywords: bremsstrahlung; Monte Carlo; high-energy accelerator; collimator; beam loss; loss localization; loss distribution

\section{Introduction}

A beam collimation system is one of the most important components in a high-power hadron accelerator for reducing uncontrolled losses and residual doses around the accelerator. The Japan Proton Accelerator Research Complex (J-PARC) $3 \mathrm{GeV}$ Rapid Cycling Synchrotron (RCS) is required to provide $1 \mathrm{MW}$ pulsed protons to a spallation target for neutron production and to the $50 \mathrm{GeV}$ Main Ring [1]. In order to achieve such a high-power output while maintaining the residual doses at a hands-on-maintenance level, the losses must be localized to a restricted area. To establish such a condition, a collimation system was installed in the J-PARC RCS [2-4]. In particular, a classical two-stage collimator system was adopted for the RCS ring collimator [5-7].

The RCS ring collimation system consists of three primary collimators and five secondary collimators. On the one hand, all the primary collimators have a transverse acceptance of $324 \pi \mathrm{mm}-\mathrm{mrad}$ and a momentum acceptance of $1 \%$, and all the secondary collimator apertures are set at $400 \pi \mathrm{mm}$-mrad. On the other hand, all the other vacuum chambers have a transverse acceptance greater than $486 \pi \mathrm{mm}$-mrad and a momentum acceptance of $1 \%$. The ring collimation system was installed in the straight injection area, except for the longitudinal primary collimator that was placed near the dispersion maximum point. The locations of the collimators are shown in Figure 1. The details of the

*Corresponding author. Email: kazami@post.j-parc.jp
RCS ring collimation system are described in the literature [2-4,8,9].

This report summarizes the performance of the beam collimation system of the J-PARC RCS.

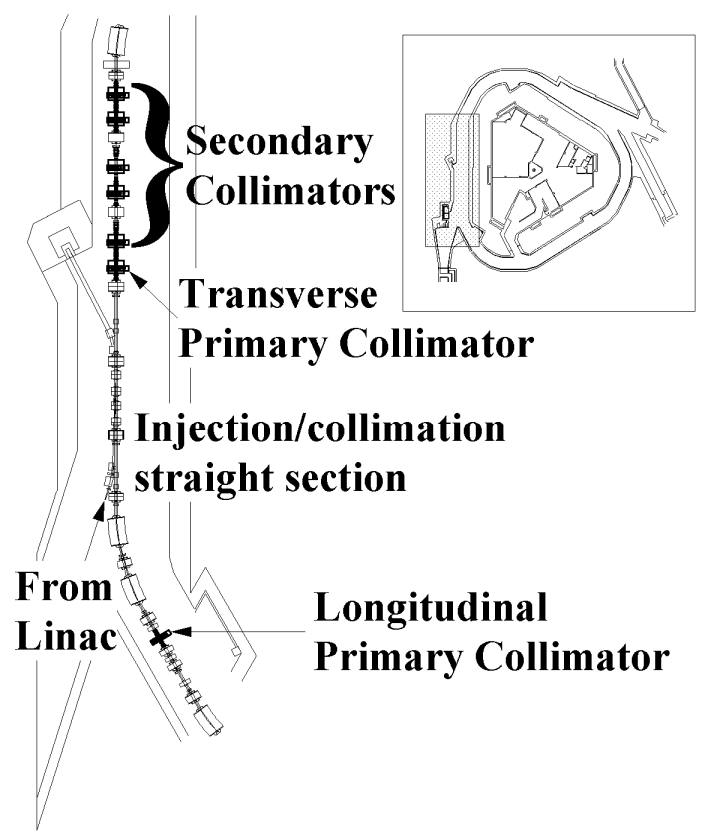

Figure 1. Locations of the collimators in the J-PARC RCS.

\section{Distribution of the losses around the ring}

The RCS continued operating with a $200-\mathrm{kW}$ output 
in May 2012, which is $20 \%$ of the design intensity [10]. The operation betatron tune was then set to $(6.42,6.42)$. With this setting, the amount of the beam loss was measured using a current transformer and was determined to be less than $1 \%$. The signals from the beam loss monitor during beam operation and the measurement results of the residual doses after operation indicate that nearly all the losses occurred within $1 \mathrm{~ms}$ after injection and they were concentrated on the transverse collimators. If there was a longitudinal halo, it was lost in the middle of the acceleration. Therefore, the longitudinal collimator was not used. The injection and extraction energies of the RCS are $181 \mathrm{MeV}$ and $3 \mathrm{GeV}$, respectively. The ramping patterns of the dipole and quadrupole magnets are sinusoidal curves. Thus, the beam energy $1 \mathrm{~ms}$ after the injection is $192 \mathrm{MeV}$. From these results, it was assumed that the amount of the loss was $1 \%$, and its energy was $200 \mathrm{MeV}$. Then the lost power was estimated to be $133 \mathrm{~W}$.

The loss distribution was calculated using the STRUCT code [11] under the above conditions. The residual dose distribution measured after beam operation and the loss distribution estimated by STRUCT are shown in Figure 2. The calculation results indicate that the beam loss is well localized on the collimators and the particles that leak from the collimator area are lost near the dispersion maximum point. This result is in agreement with the measurements, except for the $0-14$ $\mathrm{m}$ area. In the $0-14 \mathrm{~m}$ region, there were some residual doses observed that were not predicted by the calculations. These doses were caused by lost particles that were scattered by the injection foil $[12,13]$. The injection foil is needed to a charge exchange injection system which mitigates the space charge effect [14]. The foil consists mainly carbon and its thickness is typically $200 \mu \mathrm{g} / \mathrm{cm}^{2}[15]$. The scattered particles by the foil had large scattering angles and were lost before they hit the collimators. Thus, an additional collimation system was installed between the foil and the collimators. By this new collimator, the loss caused by foil scattering decreased to $<20 \%[16,17]$.

\section{Residual dose near the collimator}

The residual dose distribution in the RCS was estimated using the STRUCT and MARS codes [18] in the design stage $[19,20]$.

In this calculation, the amount of total loss was assumed to be $4 \mathrm{~kW}$. The MARS calculation indicated that the maximum dose point would be a quadrupole magnet just after the first secondary collimator, except inside the radiation shielding. The residual dose at the ceramic chamber of the quadrupole magnet was estimated to be $71.5 \mathrm{mSv} / \mathrm{h}$ after 30 days of operation and 1 day of cooling. On the other hand, the measured value was $3.6 \mathrm{mSv} / \mathrm{h}$ after 1 month of operation and $7 \mathrm{~h}$ of cooling. There was no major alteration in the

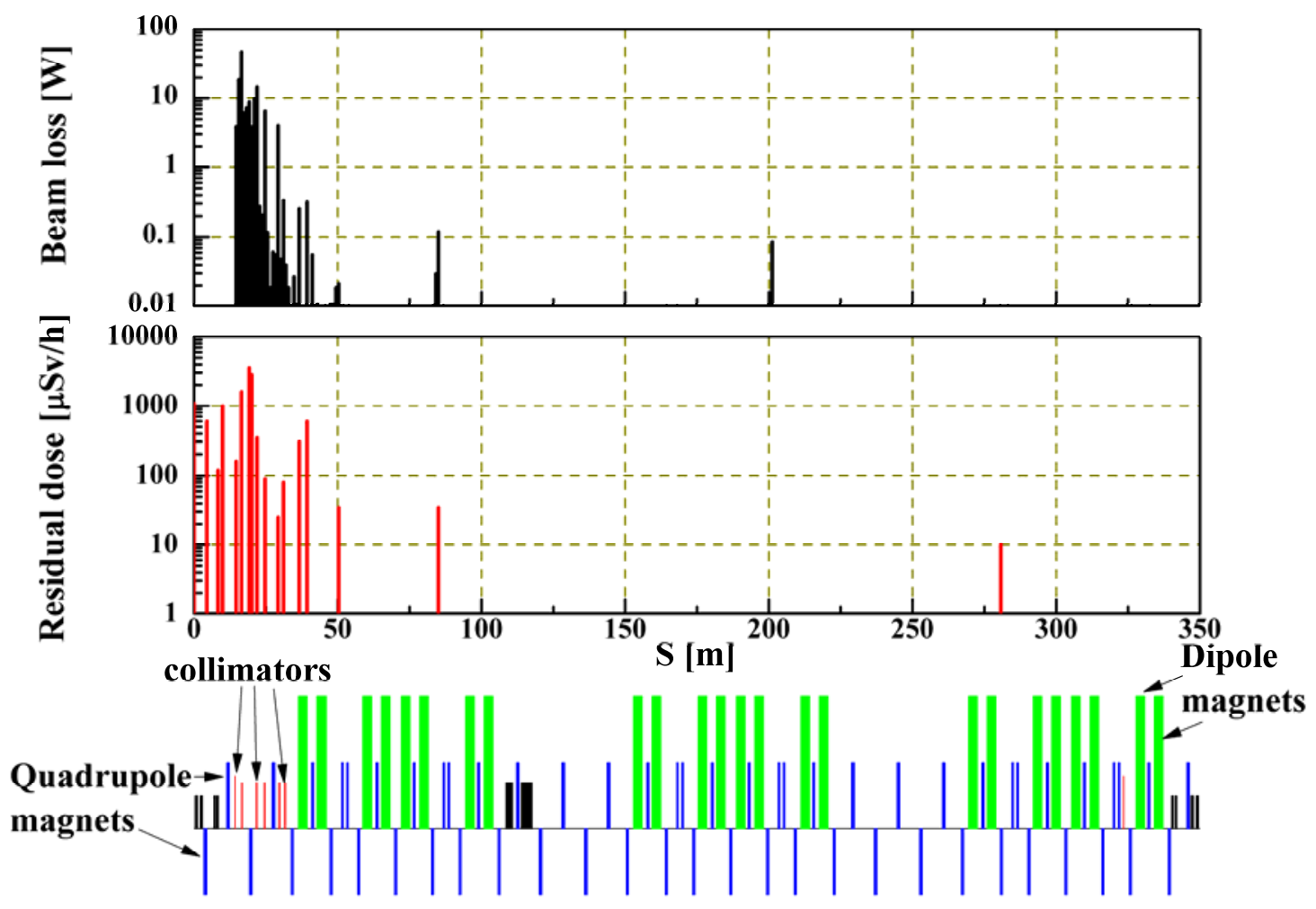

Figure 2. Comparison of the loss distribution determined with the STRUCT calculation (black) and based on the measurement results for the residual dose (red). 


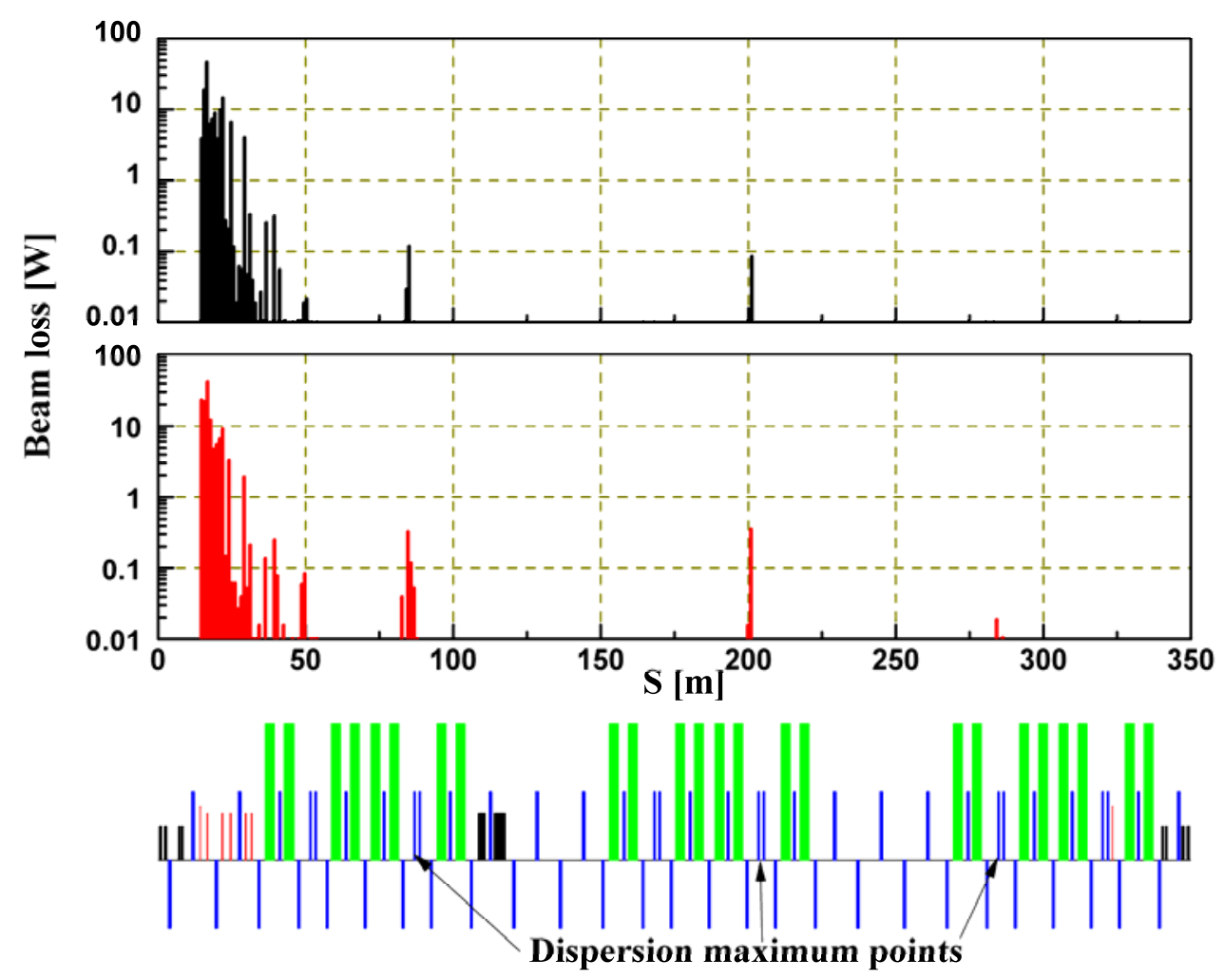

Figure 3. Arc loss dependence on the primary collimator acceptance. The black line is the loss by the primary collimator with an acceptance of $324 \pi \mathrm{mm}$-mrad, and the red line is the loss by the primary collimator with an acceptance of $240 \pi \mathrm{mm}-\mathrm{mrad}$.

beam optics, although the arrangement of the magnet changed since the MARS calculation. Therefore, the estimation agrees within a factor of 2 with the measured result, if the residual dose at the ceramic chamber is normalized by the amount of the total loss. From these results, it can be concluded that the characteristics of the residual dose are predictable with an acceptable level of accuracy using the STRUCT and MARS codes, and that the collimator design is appropriate.

\section{Arc loss due to unbalanced collimator setting}

The loss near the dispersion maximum point increased immediately after initiating $100-\mathrm{kW}$ operation [13,21]. This loss was due to the bad positioning of the primary collimator. The primary collimator position was adjusted during beam commissioning, and the horizontal primary collimator was accidentally inserted $10 \mathrm{~mm}$ too far; this position corresponded to an acceptance of $240 \pi$ mm-mrad. Figure 3 shows the calculated results using the STRUCT code with the horizontal primary collimator positioned at $240 \pi \mathrm{mm}-\mathrm{mrad}$ and $324 \pi \mathrm{mm}$-mrad acceptances. In these calculations, the secondary collimators were assumed to have a $400 \pi \mathrm{mm}$-mrad acceptance. It was found that the loss near the dispersion maximum point increased when the acceptance of the primary collimator fell below $324 \pi$ mm-mrad.
There are several possible reasons for this relationship. A particle that is scattered by the primary collimator has not only an increased transverse emittance but also a decreased energy. After scattering, particles with more than $400 \pi \mathrm{mm}$-mrad emittance would immediately be absorbed by the secondary collimator. Because there is no dispersion at the straight section, particles that have an emittance between $324 \pi \mathrm{mm}-\mathrm{mrad}$ and $400 \pi \mathrm{mm}$-mrad escape from the secondary collimators, even if they have lost a large amount of energy. Among these, the only particles that lose a large amount of energy are lost near the dispersion maximum point. If the energy loss is small, the particles circulate the synchrotron and hit the primary collimator again. On the other hand, particles that have an emittance between $240 \pi \mathrm{mm}$-mrad and $400 \pi \mathrm{mm}$-mrad can escape from the secondary collimators when the primary collimator position is $240 \pi \mathrm{mm}$-mrad. In this case, the number of particles that were scattered only a little but lost a large amount of energy was increased. Consequently, the amount of the loss near the dispersion maximum point was increased. Therefore, it was confirmed by both the experimental data and the calculated results that the collimation efficiency decreases when the balance of the collimator acceptances changes. 


\section{Conclusion}

A collimator system in the RCS has been studied as a countermeasure to the beam loss since the first design stage. The collimator system design performance (loss localization efficiency and shielding performance) after beam commissioning and operation was confirmed.

However, there were unpredictable losses before the collimation area. These losses were caused by the large angle scattering of the injection and circulating beams at the foil. These scattered particles were lost upstream of the collimator just after scattering, and therefore could not reach the collimator. Thus, an additional collimator was installed upstream of the original collimation system. Except for the upstream loss of the collimator, the beam loss distribution is in agreement with the STRUCT calculation results. Because nearly all the losses are localized on the collimators and the amount of the loss is less than $5 \%$ of the collimator capacity, the residual dose values are less than $10 \mathrm{mSv} / \mathrm{h}$ in most areas. Therefore, the RCS components except the collimators can be freely accessed.

Accurate estimation of the beam behavior was established by including detailed characteristics for each accelerator component in the simulations, which in turn enabled such very low loss operation[22,23].

\section{References}

[1] Accelerator Technical Design Report for J-PARC edited by Y. Yamazaki, KEK-Report 2002-13.

[2] K. Yamamoto, M. Kinsho, F. Noda and Y. Irie, Beam collimator design for the $3 \mathrm{GeV}$ synchrotron of the JAERI-KEK joint project, Proc. PAC01(2001).

[3] K. Yamamoto, The beam collimator system of J-PARC rapid cycling synchrotron, in Proc. $42^{\text {nd }}$ ICFA Advanced Beam Dynamics Workshop, HB2008, (2008).

[4] K. Yamamoto, Phys. Rev. ST-AB 11, 123501 (2008).

[5] J. B. Jeanneret, Phys. Rev. ST-AB 1, 081001 (1998).

[6] P. J. Bryant, CERN Accelerator School: 5th General Accelerator Physics Course, CERN 94-01 1, 159 (1994).

[7] M. Seidel, The Proton Collimation System of HERA, DESY 94-103 (1994).

[8] K. Yamamoto and M. Kinsho, Development of the collimator system for the $3 \mathrm{GeV}$ rapid cycling synchrotron, in Proceedings of the 2005 Particle Accelerator Conference (PAC05), Knoxville, (2005).

[9] K. Yamamoto, M. Okazaki, Y. Hirooka, Y. Takeuchi, A. Nakamura, H. Hanaue and M. Abe, Present status of beam collimation system of J-PARC RCS, in Proceedings of the 10th Biennial European Particle Accelerator Conference (EPAC06), Edinburgh, (2006).

$[10] \mathrm{K}$. Yamamoto, to be published in Proc. ICFA
Advanced Beam Dynamics Workshop HB2012, (2012).

[11]A. Drozhdin and N. V. Mokhov, The STRUCT Program User's Reference Manual, SSCL-MAN-0034 (1994).

[12]P. K. Saha, in Proceedings of the 46th ICFA Advanced Beam Dynamics Workshop (HB2010), Morschach, Switzerland, (2010).

[13]K. Yamamoto, in Proceedings of the 46th ICFA Advanced Beam Dynamics Workshop (HB2010), Morschach, Switzerland, (2010).

[14]H. Harada, Painting-injection Study Using a Virtual Accelerator in a High-intensity Proton Accelerator, KEK Report. No.2009-7.

[15]P. K. Saha, S. Hatakeyama, K. Yamamoto, M. Yoshimoto, H. Harada, H. Hotchi, Y. Shobuda, M. Kinsho, K. Satou and Y. Irie, State of the art online monitoring system for the waste beam in the rapid cycling synchrotron of the Japan Proton Accelerator Research Complex, Phys. Rev. ST-AB 14, 072801 (2011).

[16]K. Yamamoto, Y. Yamazaki, M. Yoshimoto, J. Kamiya, H. Harada, P. K. Saha, H. Hotchi, M. Kinsho and S. Kato, Foil scattering loss mitigation by the additional collimation system of J-PARC RCS, Proc. IPAC11, TUPS033.

[17] S. Kato, H. Harada, S. Hatakeyama, J. Kamiya, M. Kinsho, K. Yamamoto, Y. Yamazaki and M. Yoshimoto, Localization of the large angle foil scattering beam loss caused by multi-turn charge-exchange injection, in Proc. IPAC12, MOPPD074.

[18]N. V. Mokhov, The Mars Code System User's Guide, Fermilab-FN-628 (1995).

[19]N. Nakao, N. V. Mokhov, K. Yamamoto, Y. Irie and A. Drozhdin, MARS14 Shielding Calculation for the J-PARC 3GeV RCS, KEK Report2004-1, June, (2004).

[20]N. Nakao, N. V. Mokhov, K. Yamamoto, Y. Irie and A. Drozhdin, Radiation Protection Dosimetry 116, No.1-4, 85 (2005).

[21]K. Yamamoto, H. Hotchi, H. Harada, N. Hayashi, M. Kinsho, P. K. Saha, F. Tamura, M. Yamamoto, M. Yoshimoto, Y. Nakane, K. Seki and Y. Miyamoto, Beam power and residual dose history of J-PARC RCS, to be published in Proc. ICRS12.

[22]H. Hotchi, M. Kinsho, K. Hasegawa et al., Beam commissioning of the $3-\mathrm{GeV}$ rapid cycling synchrotron of the Japan Proton Accelerator Research Complex, Phys. Rev. ST-AB 12, 040402 (2009).

[23]H. Hotchi, H. Harada, N. Hayashi, M. Kinsho, P. K. Saha, Y. Shobuda, F. Tamura, K. Yamamoto, M. Yamamoto, M. Yoshimoto and Y. Irie, Beam loss reduction by a painting injection in the $3-\mathrm{GeV}$ rapid cycling synchrotron of the Japan Proton Accelerator Research Complex, Phys. Rev. ST-AB 15, 040402 (2012). 